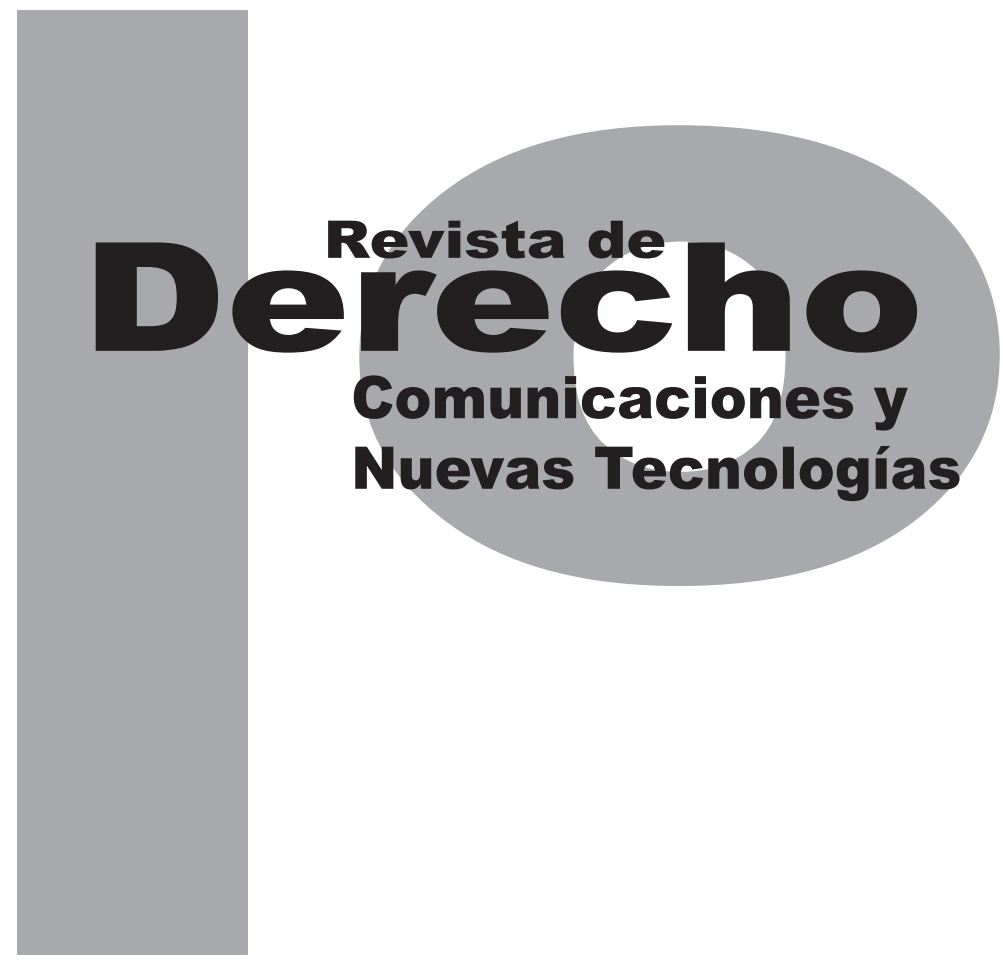

\title{
ÓRBITAS TERRESTRES: CLASES, ASPECTOS TÉCNICOS Y JURÍDICOS
}

\author{
Silvana Gómez Castillo
}

Universidad de los Andes

Facultad de Derecho

Revista de Derecho, Comunicaciones y Nuevas Tecnologías

N. ${ }^{\circ}$, Junio de 2013. ISSN 1909-7786 


\title{
Órbitas terrestres: clases, aspectos técnicos y jurídicos
}

\author{
Silvana Gómez Castillo*
}

\section{RESUMEN}

En el presente trabajo se argumenta la imposibilidad de realizar reclamos de soberanía sobre la órbita de los satélites geoestacionarios y se analizan las posiciones de los países ecuatoriales en especial Colombia y Ecuador frente a este tema, así como los tratados y convenios internacionales, además de declaraciones realizadas por distintos Estados que originaron el problema de la soberanía sobre esta órbita única en su especie.

En un primer momento, y para entender la importancia de la órbita de los satélites geoestacionarios, se exponen los tipos de órbita y sus respectivas características centrándonos en las órbitas terrestres, aspectos básicos para comprender sus fundamentos técnicos y su utilidad para la humanidad; posteriormente se aborda el tema de las regulaciones jurídicas atinentes a la soberanía sobre la órbita geoestacionaria y se

\section{AbStract}

This paper argues the impossibility of claims of sovereignty over the Geostationary Orbit, therefore, it analyzes the positions of the equatorial countries especially Colombia and Ecuador face this issue as well as international treaties and conventions and statements made by various states generating the problem of sovereignty over this orbit one of a kind. 
plantean algunas propuestas para el uso de las órbitas en general.

Palabras clave: órbita, geocéntrico, geosincrónico, geoestacionaria, Molniya, tundra, inclinación, ecuatorial, Hohmann, recurso órbita-espectro, soberanía, delimitación.
At first and for understand the importance of Geostationary orbit, this paper exposes orbit types and their respective characteristics focusing on the Earth orbits, seeking the understanding of its usefulness for humanity and understand the technical basis for subsequently achieving the correct formulation of legal regulations pertaining to sovereignty over it and considering certain proposals for the use of the orbits in general.

KEYWORDS: orbit, geocentric, geosynchronous, geostationary, Molniya, tundra, tilt, equatorial, Hohmann orbit-spectrum, sovereignty, delimitation. 


\section{SUMARIO}

Introducción - I. LAS ÓRBITAS - A. Definición - B. Colocar un objeto en órbita -C. Elementos de las órbitas - II. TIPOS DE ÓRBITA - III. ÓRBITAS TERRESTRES, ASPECTOS TÉCNICOS Y USO - A. Por sU

forma - 1. Órbita circular - 2. Órbita elíptica - 2.1 Órbita de transferencia de Hohmann - 2.2 Órbita Molniya - 2.3 Órbita Tundra - 3. Órbita semisíncrona - 4. Órbita geosíncrona - B. Por inclinación respecto al plano ecuatorial de la tierra - 1. Órbita ecuatorial - 2. Órbita polar - 3. Órbita directa 4. Órbita indirecta - C. Por altitud - 1. Órbita baja terrestre - 2. Órbita media terrestre - 3. Órbita geoestacionaria o de los satélites geoestacionarios - 4. Órbita alta terrestre - IV. SINGULARIDAD DE LA ÓRBITA DE LOS SATÉLITES GEOESTACIONARIOS - A. Aspectos técnicos - 1. Fuerzas que actúan sobre los satélites en órbita geoestacionaria - 1.1. Propulsión de lanzamiento y la propulsión de mantenimiento - 1.2. Atracción de la masa total de la Tierra - 1.3. El achatamiento de la Tierra - 1.4. La forma elíptica del Ecuador - 1.5. Atracción de la Luna y el Sol - 1.6. La presión de la radiación solar - B. Utilidad - C. Restricciones físicas de los satélites geoestacionarios - D. Las órbitas generalizadas - E. El recurso órbita-espectro (ROE) - V. MARCO JURÍDICO Y EL PROBLEMA DE LA SOBERANÍA - A. La falta de delimitación del espacio aéreo y la costumbre internacional - B. La Declaración de Bogotá - C. Las constituciones de Ecuador y Colombia - D. Pronunciamientos de la Corte Constitucional colombiana - E. El Tratado de 1967 y los principios de No Apropiación y Cooperación Internacional - F. La Convención de Viena de 1969 -VI. PROPUESTA PARA EL USO DE LAS ÓRBITAS - VII. CONCLUSIONES -Bibliografía. 


\section{Introducción}

El espacio ultraterrestre es, a la vez, un medio excepcionalmente hostil e igualmente benigno. El vacío del espacio presenta desafíos insólitos, tanto para la experimentación como para el funcionamiento de objetos localizados en él; además, a través de los numerosos satélites artificiales que existen actualmente brinda una amplia gama de aplicaciones donde las más conocidas son, sin duda, las meteorológicas y las telecomunicaciones. Las primeras proveen pronósticos e información para la prevención de desastres climatológicos. Las segundas permiten transmitir señales de radio y televisión entre uno y otro lado de los océanos y su aplicación ha aumentado exponencialmente al incrementar su capacidad y al crear redes de satélites con grandes coberturas para transmitir, por ejemplo, múltiples programas de televisión (Trench, 2011, p. 6).

El primer satélite activo, el Sputnik I fue lanzado por la URSS el 4 de octubre de 1957. Luego vino el lanzamiento del Project Score, primer satélite de comunicaciones, el 18 de diciembre de 1958 , con una potencia de 8 Watts y frecuencia $122 \mathrm{MHz}$; sus baterías operaron solo doce días. En 1960 se lanzó el primer satélite (couvier) activo de comunicaciones en órbita no geosincrónica durante 17 días. A este le siguieron los proyectos Telstar 1 (1962) con capacidad de 600 canales telefónicos y uno de televisión, el primero en recibir y emitir señales simultáneamente fue el Relay, y el Syncom que fue el primero en órbita geosincrónica en plano ecuatorial utilizado en la transmisión de los juegos olímpicos de Tokyo en 1964 (Ramírez, 1985).
El desarrollo de la investigación sobre el espacio ultraterrestre y sus aplicaciones ha sido posible gracias al constante perfeccionamiento de los sistemas de lanzamiento disponibles. Existen dos tipos de sistemas (Naciones Unidas, 1994):

a) Los sistemas de transporte reutilizables, cuya función fundamental es garantizar los vuelos tripulados y conservar las infraestructuras en órbita; su seguridad debe ser la mayor posible, habida cuenta de la presencia de seres humanos a bordo.

b) Los sistemas de lanzamiento desechables que, según su capacidad en términos de empuje, pueden poner en diferentes órbitas cargas útiles de diferente masa.

Los países se pueden clasificar en tres categorías según su capacidad espacial. Hasta el presente solo Estados Unidos y Rusia poseen la gama completa de pequeños y grandes vehículos de lanzamiento de naves espaciales tripuladas y no tripuladas, y la tecnología espacial civil y militar que es posible alcanzar en la actualidad, lo cual los coloca en la primera categoría. (Naciones Unidas, 1994).

En la segunda está un creciente número de Estados que tienen capacidad parcial para acceder al espacio: instalaciones para lanzamientos y conocimientos en materia de diseño, manufactura y funcionamiento de satélites de investigación y de otra índole. De la tercera categoría hace parte el resto de países (la gran mayoría) que no son potencias espaciales y obtienen beneficios de la explotación del espacio únicamente por medio de la capacidad de los demás (Naciones Unidas, 1994). 
Estas diferencias en cuanto a la posibilidad de acceso al espacio han llevado a algunos países a reclamar segmentos de la órbita de los satélites geoestacionarios con el fin de asegurar una posición beneficiosa en caso de llegar a tener la tecnología suficiente para acceder a ella. En este trabajo se analizarán los diferentes tipos de órbita para comprender la singularidad de la órbita de los satélites geoestacionarios, se discutirá si es posible realizar reivindicaciones de soberanía sobre esta y se propondrán usos de las órbitas que estén de acuerdo con los principios del Tratado de 1967 y otros instrumentos internacionales.

\section{LAS ÓRBITAS}

\section{A. Definición}

Por órbita se entiende la trayectoria que describe un objeto con relación a un sistema de referencia especificado, el centro de gravedad de un satélite o de otro objeto espacial por la acción única de fuerzas naturales, fundamentalmente por la de gravitación (Ramírez, 1985).

Se entiende igualmente por esta, el trayecto que describe el centro de gravedad de un objeto espacial sometido a la acción de fuerzas naturales, a las que eventualmente vienen a agregarse acciones correctivas, de poca energía, ejercidas por un dispositivo de propulsión con el objeto de lograr y mantener la trayectoria deseada.

Es así que pueden identificarse elementos comunes para la definición de órbita: que es una trayectoria del centro de gravedad de un obje- to espacial por la acción de fuerzas naturales y eventualmente artificiales con el fin de mantener el recorrido esperado.

\section{B. Colocar un objeto en órbita}

Un satélite artificial es un cuerpo cualquiera que, lanzado desde la Tierra, con una determinada velocidad y a una altura elegida, comienza a girar alrededor de nuestro planeta, siguiendo un recorrido que llamamos "órbita satelitaria" (Rubio, 1958).

A diferencia de los satélites naturales, los artificiales son construidos, controlados y lanzados por el hombre. Por causa de la atracción terrestre (fuerza de gravedad), un satélite artificial solo puede ser puesto en órbita mediante un vehículo que lo lleve allí. Una vez dejan de funcionar los motores de reacción, la continuación del movimiento del satélite artificial se rige por las leyes de la mecánica celeste (Ramírez, 1985).

Para trasladar el satélite al espacio solo existe un medio: el cohete, único vehículo concebido para moverse en un ambiente sin aire y operar en varias fases, cada una provista de su grupo propulsor y de sus reservas de combustible. Una vez que una fase se agota esta se desprende y cae, al tiempo que entra en acción la fase siguiente. Impulsado por sus diferentes fases, el cohete, con el satélite acoplado en su cono de proa, gana velocidad mientras su trayectoria se hace más horizontal. Al final, propulsado vuela ya casi paralelo a la Tierra y si ya ha alcanzado la altura necesaria, podrá apagar sus motores y permanecer en órbita (Salvat, 1974). Una vez 
dejan de funcionar los motores de reacción, la continuación del movimiento del satélite artificial se rige por las leyes de la mecánica celeste (Ramírez, 1985).

La velocidad crítica de 11,2 km, por la cual un objeto no regresaría a la tierra, se denomina velocidad de escape. En teoría, un vehículo dirigido a la Luna, a otro cuerpo celeste o a orbitar la Tierra, ha de partir impulsado por lo menos a esa velocidad (Salvat, 1974). Esta velocidad no es un valor fijo, puesto que en un disparo efectuado a $200 \mathrm{~km}$ de altura, basta con alcanzar $11 \mathrm{~km} / \mathrm{seg}$ para garantizar que la nave no retornará.

Un satélite permanece en su órbita porque su peso está exactamente compensado por la fuerza centrífuga que actúa sobre él cuando gira alrededor de la Tierra (Salvat, 1974). La velocidad v que debe tener un satélite para mantenerse en órbita circular a una altura $h$ sobre la superficie terrestre es:

$$
V=\sqrt{\frac{G * M}{R+h}} \sqrt{\frac{G * M}{R+h}}
$$

donde $\mathrm{R}$ y $\mathrm{M}$ representan, respectivamente, el radio y la masa de la Tierra y G es la llamada constante de la gravitación universal. Es aquí donde se encuentra el primer resultado interesante: la velocidad que se debe comunicar al vehículo para mantenerlo en órbita depende de la altura de vuelo, pero no de su masa. Un satélite de 200 gramos deberá ir a la misma velocidad que una nave de 100 toneladas situada a igual altura.
En la práctica, el límite de altura orbital se alcanza a 1,800,000 km sobre la Tierra; a partir de este punto la atracción solar es predominante y el objeto espacial comienza a orbitar el Sol. Si en el momento de entrar en órbita terrestre la componente horizontal de la velocidad del satélite es superior a la mínima requerida se genera una órbita elíptica. En general, puede decirse que la trayectoria será tanto más alargada cuanto mayor sea el exceso de velocidad adquirido, al límite de convertirse en una parábola de escape (Salvat, 1974).

\section{Elementos de las órbitas}

El punto más elevado de la órbita se llama apoápsis y el más bajo periápsis (en el caso particular de la Tierra, apogeo y perigeo respectivamente). Para conocer la trayectoria de un objeto espacial o su órbita es necesario distinguir cuatro parámetros: apogeo, perigeo, periodo orbital e inclinación con respecto a un plano de referencia, generalmente el Ecuador (Salvat 1974).

El periodo orbital de un satélite es el tiempo que este tarda en efectuar una revolución completa. Hay dos maneras de medirlo:

1. Respecto a la órbita en sí: tiempo invertido en recorrer toda la elipse, de perigeo a apogeo.

2. Con respecto a una referencia fija en la superficie terrestre: por ejemplo, el tiempo que tarda en cruzar dos veces consecutivas sobre el mismo meridiano.

En el primer caso se trata de un sistema fijo; en el segundo es móvil, ya que en el tiempo que dura una revolución del satélite la Tierra tam- 
bién ha girado cierto ángulo, arrastrando consigo al meridiano de referencia. Para diferenciar ambos métodos se habla de periodo orbital y periodo de revolución, respectivamente.

La inclinación de la órbita no es otra cosa que el ángulo que forma su plano con el plano del Ecuador. Cuando este ángulo es cero (0) ambos coinciden y se habla de trayectorias ecuatoriales; cuando es de $90^{\circ}$, de trayectorias polares, puesto que sobrevuela ambos polos terrestres. A veces algunos satélites se disparan con inclinaciones superiores a los $90^{\circ}$ y entonces su movimiento tiene lugar en sentido contrario a la rotación de la Tierra, de ellos se dice que siguen trayectorias retrógradas.

Manejando adecuadamente los parámetros orbitales puede conseguirse poner el satélite en órbita geoestacionaria: a una órbita circular a $35,900 \mathrm{~km}$ de altura le corresponde un periodo orbital de $23 \mathrm{~h} 56 \mathrm{~min} 4 \mathrm{~s}$, el mismo que el de la rotación de nuestro planeta. Cualquier satélite insertado en esta trayectoria girará a igual velocidad que la Tierra, manteniéndose siempre fijo sobre el mismo meridiano. Solo fluctuará en latitud, de forma que para un observador fijo en el suelo, parecerá describir en el firmamento una especie de " 8 ", tanto más alargado cuanto más acusada sea su inclinación con respecto al Ecuador. Si esa órbita circular es, además, ecuatorial, el "8" quedará reducido a un punto. El satélite permanecerá entonces "fijo" tanto en longitud como en latitud: estará "anclado" en un lugar del espacio y nunca se apartará de ahí. Las antenas de las estaciones de seguimiento solo tendrán que ser apuntadas una vez, al principio, con la seguridad de encontrar al satélite siempre en el mismo punto (Salvat, 1974), fenómeno que se da únicamente en este punto del espacio y del cual se hablará con mayor profundidad más adelante.

\section{TIPOS DE ÓRBITA}

Existen múltiples tipos de órbita clasificados según el cuerpo al que orbitan, según su inclinación, su forma, su uso. En el presente trabajo se tratarán solo aquellas órbitas que por ser utilizadas por el hombre para el posicionamiento de satélites en torno a la Tierra, son de interés para los estudiosos del derecho espacial.

\section{III. ÓRBITAS TERRESTRES, ASPECTOS TÉCNICOS Y USO}

Por su forma

\section{1. Órbita circular}

En esta órbita el objeto gira y describe un círculo; la velocidad de giro es una sola y el radio de órbita es determinado; la atracción por gravedad se denomina centrípeta.

$$
v=\sqrt{\frac{M}{r}}
$$

\section{2. Órbita elíptica}

Una órbita elíptica tiene una excentricidad mayor que cero y menor que uno (si posee excentricidad 0 es una órbita circular y con excentricidad 1 es una órbita parabólica). La energía específica de una órbita elíptica es negativa. 
Ejemplos de órbitas elípticas son: órbita de transferencia Hohmann (ejecutada cuando un satélite cambia la cota de giro orbital), órbita Molniya y órbita tundra.

Bajo las suposiciones estándar en astrodinámica la velocidad orbital $(v)$ de un cuerpo que describe una trayectoria sobre una órbita elíptica se puede calcular como:

$$
v=\sqrt{2 \mu\left(\frac{1}{r}-\frac{1}{2 a}\right)}
$$

donde:

$\mu$ es un parámetro gravitacional estándar, $r$ es la distancia radial desde el cuerpo orbitante al cuerpo central, y $a$ es la longitud del semieje mayor de la elipse.

El periodo orbital (T) de un cuerpo que viaja sobre una trayectoria elíptica puede ser calculado mediante la siguiente fórmula:

$$
T=\frac{2 \pi}{\sqrt{\mu}} a^{\frac{3}{2}}
$$

donde $\mu$ es un parámetro gravitacional estándar y $a$ es la longitud del semieje mayor de la elipse.

\section{1. Órbita de transferencia de Hohmann}

Como su nombre lo indica es una trayectoria de transferencia; es usada para pasar de una órbita a otra más alta con menor gasto de combustible que si se llegara directamente por la propulsión del vehículo. Corresponde a una mitad de una órbita elíptica que toca, tanto la órbita ini- cial que se desea dejar como la órbita final que se quiere alcanzar. La órbita de transferencia se inicia disparando el motor de la nave espacial para acelerarla creando una órbita elíptica; esto añade energía a la órbita de la nave espacial. Cuando la nave alcanza la órbita final se debe acelerar de nuevo el motor para alcanzar la velocidad necesaria para hacer una nueva órbita circular. Pero ¿cómo se logra esto?, veamos (Franco, 2010):

\section{Órbita circular interior}

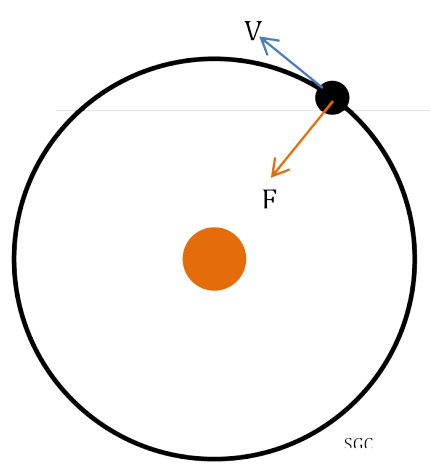

Cuando la nave espacial describe una órbita circular de radio $r_{A}$, el módulo de la velocidad $v_{A}$ se puede calcular aplicando la dinámica del movimiento circular uniforme

$$
\frac{G M m}{r_{A}^{2}}=m \frac{v_{A}^{2}}{r_{A}} \quad v_{A}^{2}=\frac{G M}{r_{A}}
$$

donde $M$ es la masa de la Tierra, $G$ es la constante de la gravitación universal, y $m$ es la masa de la nave que se simplifica en las ecuaciones del movimiento.

La energía $E_{1}$ de la nave espacial en la órbita circular inicial es la mitad de la energía potencial 


$$
E_{1}=\frac{1}{2} m v_{A}^{2}-\frac{G M m}{r_{A}}=-\frac{G M m}{2 r_{A}}
$$

\section{Órbita semielíptica de transferencia}

Para calcular la velocidad que debe llevar la nave espacial en el punto A para que alcance la órbita exterior en B, basta aplicar las propiedades central y conservativa de la fuerza de atracción.

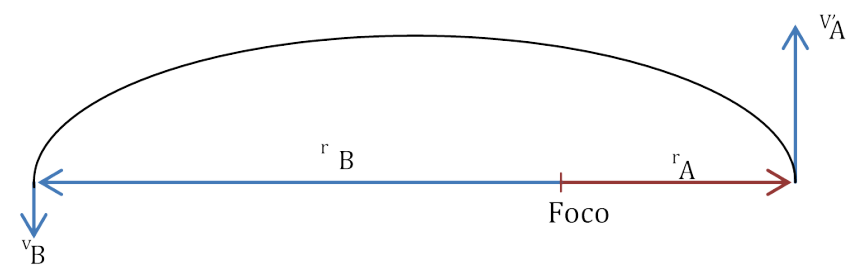

Por la propiedad de la fuerza central, el momento angular es constante y, por tanto, tiene el mismo valor en $A$ que en $B$ :

$$
m r_{A} v^{\prime}=m r_{B} v_{B}
$$

Por la propiedad de fuerza conservativa la energía es constante en todos los puntos de la trayectoria, y en particular es la misma en A que en $B$.

$$
\frac{1}{2} m v_{A}^{2}-\frac{G M m}{r_{A}}=\frac{1}{2} m v_{B}^{2}-\frac{G M m}{r_{B}}
$$

Conocidos $r_{A}$ y $r_{B}$ podemos calcular en este par de ecuaciones las incógnitas $v_{A}^{\prime}$ y $v_{B^{*}}$.

$v_{A}^{\prime 2}=\frac{2 G M r_{B}}{r_{A}\left(r_{A}+r_{B}\right)} \quad v_{B}^{2}=\frac{2 G M r_{A}}{r_{B}\left(r_{A}+r_{B}\right)}$

La energía de la nave espacial es constante en todos los puntos de la trayectoria e igual a

$$
E_{2}=\frac{1}{2} m v_{A}^{\prime 2}-\frac{G M m}{r_{A}}
$$

La energía que hemos de suministrar al satélite en la posición A para que pase de la órbita circular a la trayectoria de transferencia es la diferencia $E_{2}-E_{1}$ o bien,

$$
\Delta E_{A}=\frac{1}{2} m v_{A}^{2}-\frac{1}{2} m v_{A}^{2}=\frac{G M m}{2 r_{A}}\left(\frac{r_{B}-r_{A}}{r_{B}+r_{A}}\right)
$$

\section{Órbita circular exterior}

Una vez que la nave espacial llega al punto $B$ ha de cambiar su velocidad para seguir la trayectoria circular de radio $r_{B}$. De nuevo, aplicando la dinámica del movimiento circular uniforme tenemos:

$$
\frac{G M m}{r_{B}^{2}}=m \frac{v_{B}^{2}}{r_{B}} \quad v_{B}^{2}=\frac{G M}{r_{B}}
$$

La energía $E_{3}$ de la nave espacial en la órbita circular final es:

$$
E_{3}=\frac{1}{2} m v_{B}^{2}-\frac{G M m}{r_{B}}=-\frac{G M m}{2 r_{E}}
$$

La energía que hemos de suministrar al satélite para que pase de la órbita de transferencia elíptica a la órbita circular de radio $r_{B}$ es la diferencia $E_{3}-E_{2}$ o bien,

$$
\Delta E_{B}=\frac{1}{2} m v_{B}^{2}-\frac{1}{2} m v_{B}^{2}=\frac{G M m}{2 r_{B}}\left(\frac{r_{B}-r_{A}}{r_{B}+r_{A}}\right)
$$

El tiempo que tarda la nave espacial en pasar del punto $A$ al punto $B$, principio y fin de la tra- 
yectoria de transferencia, es la mitad del periodo $P$

$$
P^{2}=\frac{4 \pi^{2} a^{3}}{G M} \quad a=\frac{r_{A}+r_{B}}{2}
$$

siendo a el semieje mayor de la elipse.

\section{2. Órbita Molniya}

Las órbitas Molniya son un subgrupo de las órbitas semisincrónicas, que son sumamente elípticas, caracterizadas por puntos bajos (perigeo) de unos cientos de kilómetros, y puntos altos (apogeos) de casi 40.000 kilómetros. Normalmente estas órbitas tienen un ángulo de 63 grados y se usan para abarcar las regiones polares de latitud alta (Naciones Unidas, 1994).

\section{3. Órbita tundra}

Es una órbita muy excéntrica con una inclinación de $63,4^{\circ}$ y un periodo orbital igual a un día sideral (23h, 56m, 4s).

\section{3. Órbita semisíncrona}

Las órbitas semisincrónicas se caracterizan por un periodo de 12 horas, con satélites a una altura de 20.000 kilómetros. Las órbitas semisincrónicas circulares son las que normalmente recorren los satélites de navegación modernos (Naciones Unidas, 1994). Son muy empleadas por la Unión Soviética en su programa de satélites meteorológicos y de telecomunicaciones.

\section{4. Órbita geosíncrona}

Es una órbita donde el satélite tiene un periodo igual al periodo de rotación de la Tierra y en la misma dirección. Un cuerpo en una órbita síncrona no ecuatorial aparecerá oscilante de norte en sur en torno a un punto sobre el Ecuador del planeta, mientras que un cuerpo en una órbita elíptica parecerá que oscila de este a oeste. Para un observador situado en el cuerpo orbitado o central, la combinación de estos dos movimientos produce una figura en forma de 8. $\mathrm{Si}$, además, esta es ecuatorial y circular se llama órbita geoestacionaria.

\section{B. Por inclinación respecto al plano ecuatorial de la Tierra}

De acuerdo con Velandia (2012), estas son:

\section{1. Órbita ecuatorial}

Es la que se encuentra con una inclinación de $0^{\circ}$

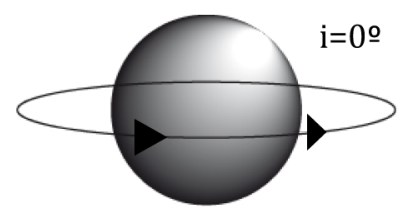

\section{2. Órbita polar}

Recorren 16 órbitas por día y son típicas de Ios satélites militares de reconocimiento, dado que cada día, a la misma hora, sobrevuelan los mismos puntos del globo. El único límite viene impuesto por la imaginación del analista de trayectorias (Salvat, 1974). 


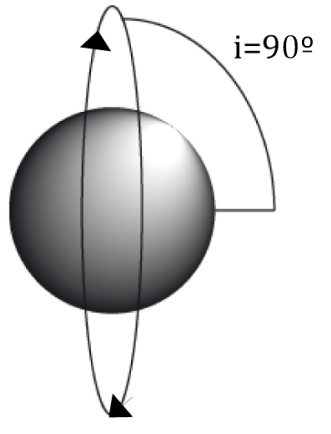

3. Órbita directa

Tiene una inclinación entre 0 y $90^{\circ}$

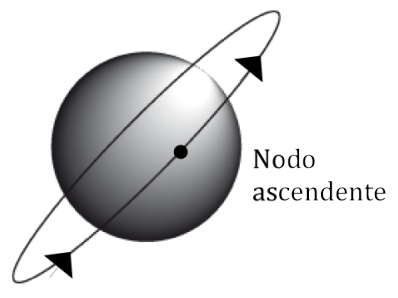

4. Órbita indirecta

Se encuentra con una inclinación entre $90^{\circ}$ y $180^{\circ}$

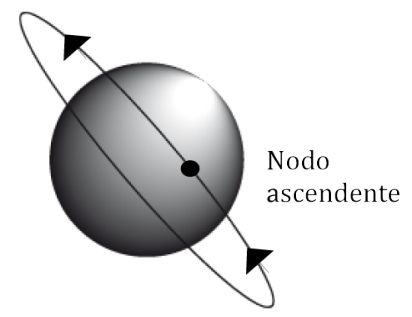

C. Por altitud

\section{1. Órbita baja terrestre}

Las órbitas terrestres bajas incluyen las órbitas que van desde unos cientos de kilómetros hasta más de 1000 kilómetros; pueden tener cualquier inclinación, aunque normalmente es alta a fin de aprovechar al máximo la cobertura de las zonas de latitud alta de la superficie terrestre (Salvat, 1974).

Los satélites encauzados en este tipo de órbitas son de tres tipos: Leo (Low Earth Orbit: órbitas bajas), мeo (Medium Earth Orbit: órbitas medias) y GEO.

LoS LEO pequeños (centenares de Kbps) están destinados a aplicaciones de bajo ancho de banda, los LEo grandes (miles de Kbps) albergan las aplicaciones de los anteriores y otras como telefonía móvil y transmisión de datos, y finalmente Ios LEO de banda ancha (megaleo) que operan en la banda de Mbps, entre los que se encuentra Teledesic. Este tipo de satélites se denomina de seguimiento, ya que necesitan antenas seguidoras de grandes dimensiones para la recogida de informaciones. Están ubicados entre 200 y $500 \mathrm{~km}$ de altura, y son utilizados por los transbordadores, los laboratorios espaciales, los satélites de observación y fotografía de la tierra o los destinados a misiones científicas o militares especiales (Trench, 2011).

La puesta en órbita de satélites LEO presenta problemas tales como:

1. Saturación de las órbitas: elevada cantidad de satélites ya existentes en esa zona y elevado número de proyectos de lanzamientos de satélites de este tipo.

2. Chatarra espacial: dificultades para la buena circulación debido a restos de otros satélites en la zona.

3. Pérdida y sustitución de satélites: cabe la posibilidad de que estos satélites caigan en 
la atmósfera al terminar su vida útil y se desintegren en esta. Además, habrá que tener en cuenta una política de sustitución de este tipo de satélites pues están expuestos a múltiples peligros, incluso antes del final de su vida útil.

4. Visibilidad del satélite: por su gran velocidad resulta difícil seguirles la pista, además, solo son visibles 18-20 minutos antes de desaparecer en el horizonte.

5. Problema de la antena: se resuelve utilizando una antena del tipo array en fase, que son dispositivos autodirigidos (antenas más pequeñas) capaces de seguir el rastro de varios satélites a la vez sin moverse físicamente, por medio de señales levemente diferentes recibidas por el conjunto de antenas. Con este tipo de antenas desaparece el problema de mantener un enlace activo cuando se pierde la visión del satélite, pues es posible tener como mínimo dos satélites a la vista en todo momento ya que el conjunto de antenas es consciente de la posición de todos los satélites e inicia un nuevo enlace antes de cortar el ya existente.

\section{2. Órbita media terrestre}

Se encuentra entre 10075 y 20150 Km de altura. A diferencia de las GEO su posición relativa respecto a la Tierra no es fija. Debido a su menor altitud se necesitarán más satélites para cubrir la superficie terrestre, pero por contra se reduce la latencia del sistema de forma significativa. En la actualidad no existen muchos MEo, y se utilizan principalmente para posicionamiento (Teledesic).

\section{3. Órbita geoestacionaria o de los satéli- tes geoestacionarios}

Pertenece a las órbitas terrestres geosincrónicas que se sitúan a una altura de casi 36.000 kilómetros: tienen un periodo de aproximadamente un día, lo cual permite al satélite captar instantáneamente casi la mitad de la superficie de la tierra. Esas órbitas son útiles para las comunicaciones, la alerta temprana o la reunión de información por medios electrónicos. Si el satélite se encuentra en el plano orbital del Ecuador de la Tierra (inclinación cero), se les llama órbitas geoestacionarias, y permiten a un solo satélite abarcar durante las 24 horas del día determinada zona (Naciones Unidas, 1994). La particularidad de estas órbitas es innegable y por ello se tratarán aparte.

\section{4. Órbita alta terrestre}

Es una órbita geocéntrica por encima de la órbita geosíncrona de 35786 km; también conocida como órbita muy excéntrica u órbita muy elíptica. Allí van a parar los satélites que se encuentran cerca de finalizar su vida útil, para evitar la congestión de la órbita geoestacionaria.

\section{SINGULARIDAD DE LA ÓRBITA DE LOS SATÉLITES GEOESTACIONARIOS}

La órbita de los satélites geoestacionarios no es solo particular por las condiciones que se verán más adelante, sino también porque fue descubierta antes de que se hubiera puesto el primer objeto espacial en órbita o que existiera la tecnología necesaria para ello. En 1945, el 
entonces oficial de radar de la RAF (Real Fuerza Aérea), Arthur C. Clarke, escribió un artículo en la revista Wirelees World, donde hablaba de la posibilidad de colocar tres repetidores separados $120^{\circ}$ entre sí a una distancia de $36.000 \mathrm{~km}$ de altura, de tal manera que se cubriera toda la Tierra y se mantuviera comunicada mediante radio comunicaciones (Peredo, 2004), dando de esta manera paso a la órbita geoestacionaria u órbita de Clarke.

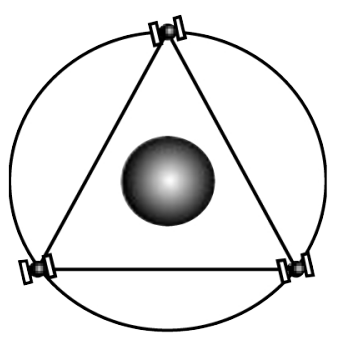

\section{A. Aspectos técnicos}

Descubierta por científicos de la talla de Kepler, Newton, Maswell y Clarke, la órbita GEo consiste en un anillo de 150 kilómetros de ancho en sentido norte-sur y un espesor de 30 kilómetros que circunda la Tierra a una altura de 35.875 $\mathrm{km}$. Su existencia depende de la relación con los fenómenos gravitacionales de la Tierra (Ramírez, 1985).

Es una órbita circular en el plano ecuatorial, en la cual el periodo de revolución sideral tiene la misma dirección e igual periodo que el de rotación de la Tierra, es decir, 23 h 56 min 4 s y no 24 h como erróneamente se ha creído. Según Naciones Unidas (1977), el valor más exacto disponible del periodo es:
$\mathrm{P}=\left(86.164,09892+0,0015^{*} \mathrm{~T}\right) \mathrm{s}$ y se expresa en minutos, lo cual es aproximadamente $1.436,0683 \mathrm{~min}$ o $23 \mathrm{~h}, 56 \mathrm{~min}, 4.1 \mathrm{~s}$.

Donde T es la época a partir de 1900.0 en siglos. Debido a la fricción dinámica, la velocidad de rotación de la tierra está disminuyendo, y el periodo crece a razón de 0,0015 segundos por siglo; en consecuencia el radio de la órbita geoestacionaria crece a razón de $51 \mathrm{~cm}$ por siglo.

De modo que un satélite es geoestacionario una vez adquiera la altura, la velocidad, la gravitación de la Tierra y sea puesto en la órbita geoestacionaria, es decir, con una inclinación de $0^{\circ}$ con respecto al Ecuador y a una altura de $35,875 \mathrm{~km}$.

\section{Fuerzas que actúan sobre los satélites en órbita geoestacionaria}

De acuerdo con Naciones Unidas (1977), el desplazamiento de un satélite ubicado en la órbita geoestacionaria está condicionado a una concatenación de factores diversos aparte de la energía transmitida o propia del vehículo, la masa y la altitud del objeto espacial y la fuerza de la gravedad de la Tierra, de la Luna y el Sol, incluso de la prestación de la radiación solar (Ramírez, 1985).

\subsection{Propulsión de lanzamiento y la propulsión de mantenimiento}

La posición inicial del satélite en la órbita es determinada por el lanzamiento y los posteriores impulsos adicionales aplicados con cohetes. Aun cuando se haya alcanzado la posición no- 
minal deseada se necesitan correcciones de mantenimiento si se desea conservar el satélite en una zona predeterminada alrededor de la posición nominal. Esas correcciones deben realizarse periódicamente durante toda la vida del satélite. De lo contrario, las fuerzas de origen natural lo desviarán fuera de la región prevista inicialmente.

Teóricamente, la propulsión de mantenimiento podría ser lo suficientemente poderosa como para mantener el satélite en posición estacionaria con respecto a la Tierra en rotación aun fuera de la órbita geoestacionaria e incluso sobre cualquier punto y a cualquier altura.

\subsection{Atracción de la masa total de la Tierra}

Es la fuerza de origen natural más importante que actúa sobre un satélite geoestacionario. La atracción a una distancia $r$ del centro de la Tierra puede calcularse a partir del periodo del satélite, usando la tercera ley de Kepler.

$$
r^{3}=\frac{G E}{4 \pi^{2}} P^{2}
$$

donde $\mathrm{GE}=398.600,5 \mathrm{~km} 3 \mathrm{~s}-2$ es la constante gravitatoria multiplicada por la masa terrestre. El valor citado anteriormente para el periodo conduce a un valor de $42.164,175 \mathrm{~km}$ para el radio de la órbita geoestacionaria.

\subsection{El achatamiento de la Tierra}

Su principal efecto es el de aumentar el radio de la órbita geoestacionaria al valor de

$$
r_{g}=42.164,697 \mathrm{~km}
$$

restando el radio ecuatorial de la Tierra

$$
r_{o}=6.378,140 \mathrm{~km}
$$

la altura nominal de la órbita geoestacionaria sobre el Ecuador es de

$$
h=35.786,557 \mathrm{~km}
$$

\subsection{La forma elíptica del Ecuador}

La diferencia en los rayos máximos y mínimos del Ecuador no supera los 70 metros, pero basta para provocar en un satélite geoestacionario importantes oscilaciones en torno del eje menor del Ecuador. Las aptitudes (variaciones de inclinación que sufren los satélites) de las oscilaciones pueden llegar a los 90 grados y su periodo supera los dos y tres años.

Los satélites derivan con respecto a la Tierra en rotación, a una velocidad de 0.4 grados por día. Si derivan hacia el Oeste se elevan hasta 34 kilómetros sobre la órbita geoestacionaria. Si derivan hacia el Este, descienden hasta 34 kilómetros por debajo de dicha órbita.

Los satélites ubicados en la prolongación del eje menor del Ecuador, situado aproximadamente en las longitudes $105^{\circ}$ y $75^{\circ} \mathrm{E}$ no resultan afectados porque están en equilibrio estable. Los satélites ubicados sobre el eje mayor, a los $15^{\circ} \mathrm{O}$ $y$ a los $165^{\circ} \mathrm{E}$, se hallan en equilibrio inestable. 


\subsection{Atracción de la Luna y el Sol}

La atracción de la Luna y el Sol, más el achatamiento de la Tierra ejercen sobre el satélite una fuerza que lo desplaza del plano ecuatorial. En consecuencia, la inclinación de un satélite, inicialmente igual a 0 , aumenta en 0.85 grados por año hasta alcanzar la inclinación máxima de 14.6 grados al cabo de 26.5 años, luego la inclinación disminuirá nuevamente a cero. Este efecto provoca también un leve cambio en la altura y ligeras oscilaciones en la longitud. La proyección de la órbita sobre la superficie terrestre tendrá la figura de un ocho (8) con la amplitud igual a la inclinación.

\subsection{La presión de la radiación solar}

Provoca una oscilación anual de excentricidad de la órbita. La magnitud del efecto depende de la superficie del satélite que se halla orientada hacia el Sol. La excentricidad aumentará durante seis meses y disminuirá el valor inicial durante el resto del año.

\section{B. Utilidad}

De todas las órbitas que circundan la Tierra esta es la única que reúne las características para fijar satélites que parecerán estáticos desde la Tierra. La ventaja de esta órbita es que un satélite geoestacionario tiene bajo observación constante una amplia zona de la Tierra y no es necesario ubicar continuamente una antena terrestre para rastrearlo.

Los satélites de televisión y telecomunicaciones se sitúan en esta órbita ya que las comunicacio- nes óptimas son las que se emplean en órbita circular o elíptica y un punto en la órbita geoestacionaria resulta excelente para la colocación de un satélite para un servicio de radiocomunicación espacial. Contrario a lo que sucede con las demás órbitas elípticas que circundan la Tierra, que no tienen el mismo sistema de rotación sideral de ella, donde un satélite puesto en una de esas órbitas solo será rastreado cuando pase por encima de la antena receptora o transmisora captando señales fragmentarias (Ramírez, 1985).

Para alcanzar la órbita geoestacionaria, el satélite se ha de situar primero en una órbita de transferencia muy elíptica cuyo perigeo está muy cerca de la Tierra (aproximadamente 200 km) y permite alcanzar la altitud definitiva en el apogeo. Aquí, el satélite podría durar indefinidamente en esa órbita pero se le aplica una aceleración mediante el encendido de los llamados motores de apogeo del satélite, de forma que su velocidad, que en ese instante es de $1.5 \mathrm{~km} / \mathrm{s}$ aumenta hasta alcanzar el valor de la velocidad circular de sincronismo: 3 km/s (CNES, 1996). (Ver órbita de transferencia de Hohmann).

\section{Restricciones físicas de los satélites geoestacionarios}

El artículo 33 inc. 2do. del Convenio Internacional de Telecomunicaciones suscrito en Málaga, en 1973, reconoce que la órbita geoestacionaria constituye un recurso natural limitado. En efecto, el mencionado artículo estipula que

Los miembros tendrán en cuenta que las frecuencias y la órbita de los satélites geoesta- 
cionarios son recursos naturales limitados que deben utilizarse en forma eficaz y económica para permitir el acceso equitativo a esta órbita y a sus frecuencias a los diferentes países o grupos de países, según sus necesidades y los medios técnicos de que dispongan, de conformidad con lo establecido en el Reglamento de Radiocomunicaciones.

Hemos visto que esta órbita es única dentro del número infinito de órbitas geosincrónicas. Tiene una inclinación de cero grados con respecto al Ecuador. Por esta razón, la órbita geoestacionaria representa el único sitio en el universo en que mediante un sistema de propulsión económicamente razonable, pueden aprovecharse los atributos naturales del lugar, para mantener, sin mayores dificultades técnicas, un satélite en posición estacionaria (Gaviria, 1978). Lo anterior explica el gran número de satélites en explotación que utilizan esa órbita y el peligro de saturación que esto representa.

No se puede afirmar con precisión cuántos satélites se pueden ubicar en la órbita geoestacionaria. Sin embargo sí se puede prever, con el desarrollo tecnológico y la desordenada ubicación de satélites en las posiciones orbitales, que la órbita puede llegar a saturarse ${ }^{1}$.

Si se dan choques entre satélites o con desechos espaciales, se originan desechos de segunda generación que significan un serio peligro para los satélites activos. Más aún, el peligro de choque con partículas pequeñas originadas por la situación anterior crea a su vez un nuevo

1 Problemática comentada en el documento "A" de la Conferencia 101/B.7. problema, puesto que dichas partículas por su tamaño pequeño difícilmente pueden ser detectadas desde la Tierra. En consecuencia, las posibilidades de choque aumentarían (Williams, 1990).

Hoy en día el fenómeno no reviste mayor gravedad, pues la tecnología ha permitido desalojar satélites de las posiciones orbitales enviándolos a órbitas altas para descongestionar los espectros de frecuencia.

\section{Las órbitas generalizadas}

Teniendo en cuenta el riesgo de saturación de la órbita geoestacionaria, las órbitas generalizadas constituyen una solución en razón de que permiten a los países lograr lo que deseaban en la geoestacionaria sin realizar mayores esfuerzos.

En el Reglamento de Radiocomunicaciones de la Unión Internacional de Telecomunicaciones $\left(\mathrm{UIT}^{2}\right)$, la órbita geoestacionaria se define por su inclinación y excentricidad nulas y por su periodo, que se supone igual al periodo de rotación de la Tierra. Las órbitas geosincrónicas comprenden a la órbita geoestacionaria como un caso especial (Naciones Unidas, 1977).

La trayectoria descrita sobre la superficie terrestre por el punto situado bajo un satélite geosin-

2 En el año de 1932 se realizó en Madrid una conferencia que decidió crear la Unión Internacional de Telecomunicaciones (UIT), una organización intergubernamental que cuenta con más de 140 países miembros y es el organismo especializado de Naciones Unidas encargado de regular las telecomunicaciones a nivel internacional, coordinando a los gobiernos y al sector privado en el desarrollo de redes y servicios. El régimen está enmarcado por algunos principios de carácter rígido como el de la igualdad de trato, el secreto de las comunicaciones y la prioridad de los servicios de seguridad. 
crónico en una órbita circular inclinada tiene la forma de un 8 centrado en el ecuador. El satélite mismo cruza el ecuador a la distancia geoestacionaria (Naciones Unidas, 1977).

Una órbita inclinada excéntrica proyecta sobre la superficie terrestre la forma de un 8 distorsionado, que va desde asimetrías imperceptibles hasta curvas en ángulo o elípticas y casi circulares. La distorsión depende de la inclinación y excentricidad y, además, de la posición del perigeo de la órbita. En general, los satélites situados en estas órbitas no pasan por el Ecuador a la distancia geoestacionaria pero permanecen cerca de esta (Naciones Unidas, 1977).

Un ejemplo para una estación terrestre en el hemisferio septentrional es el que proporciona una órbita de $2,5^{\circ}$ de inclinación, 0,05 de excentricidad y un perigeo situado a la máxima latitud meridional de la órbita. Un satélite en esta órbita atraviesa el plano ecuatorial dentro de la distancia geoestacionaria y la proyección terrestre de su trayectoria es una curva convexa que requiere movimientos de seguimiento no mayores de alrededor de $12^{\circ}$. Sería posible ubicar en la órbita diez satélites con una separación mínima de $2^{\circ}$, aumentando así significativamente la capacidad del arco pertinente de la órbita geoestacionaria (Naciones Unidas, 1977).

\section{E. El recurso órbita-espectro (ROE)}

Como se vio anteriormente, la acumulación de vehículos en el espacio puede hacer peligrosa la navegación y aumentar las posibilidades de choque, ya sea entre estos o entre ellos y aeronaves en vuelos regulares o normales por las capas inferiores del espacio cuando aquellos regresen a la Tierra.

Por esto, ya en 1964 (Estradé, 1964), se consideraba importantísimo que los lanzamientos no pudieran hacerse sin la verificación internacional y el permiso para establecimiento de órbitas. En Málaga - Torremolinos fue el primer Convenio en donde la uit se manifestó acerca de la órbita de los satélites geoestacionarios y estableció las siguientes disposiciones espaciales relativas a las radiocomunicaciones:

1. Los Miembros procurarán limitar el número de frecuencias y el espectro utilizado al mínimo indispensable para asegurar el funcionamiento satisfactorio de los servicios necesarios. A tales fines, se esforzarán por aplicar, a la mayor brevedad, los adelantos técnicos más recientes.

2. En la utilización de bandas de frecuencias para las radiocomunicaciones espaciales, los Miembros tendrán en cuenta que las frecuencias y la órbita de los satélites geoestacionarios son recursos naturales limitados que deben utilizarse en forma eficaz y económica para permitir el acceso equitativo a esta órbita y a esas frecuencias a los diferentes países o grupos de países según sus necesidades y los medios técnicos de que dispongan, de conformidad con lo establecido en el Reglamento de Radiocomunicaciones.

En Nairobi (1982) se firmó un Convenio Internacional de Telecomunicaciones auspiciado por la UIT, que en su artículo 33 reglamenta la utilización nacional del espectro de frecuencias radioeléctricas y de la órbita de los satélites geoestacionarios (Ramírez, 1985). 
De acuerdo con el reglamento de la uIT, los países que proyecten un sistema de telecomunicaciones por satélite tienen que notificar, por lo menos cinco años antes de supuesta en servicio, toda la información detallada sobre el propio sistema y sus satélites a la Junta del Reglamento de Radiocomunicaciones, la cual fue creada como parte de la nueva estructura de la UIT en 1992, y es la sucesora de la Junta Internacional de Registro de Frecuencias (IFRB), cuyos miembros trabajaban en régimen de dedicación exclusiva (Abourdaham).

Una vez notificada a los miembros de la uit la solicitud y examinada técnicamente esa notificación, se inscribe la asignación en el Registro Internacional de Frecuencias.

Todos tienen derecho a utilizar una trayectoria, a condición de no interferir en un derecho anterior en igual sentido que tuviese otro. El fundamento de este derecho se encuentra en el Tratado del Espacio de 1967 que consagra el principio de libertad de desplazamiento. Todos tienen derecho a colocar un objeto espacial en órbita pero el segundo en el tiempo deberá respetar el camino elegido por el primero (primero en el tiempo, primero en el derecho).

En el numeral 8 del documento aprobado por la Subcomisión de Asuntos Jurídicos en su 39 periodo de sesiones (A/AC.105/738, anexo III) "Algunos aspectos relativos a la utilización de la órbita geoestacionaria", se añaden al principio de "quien Ilega primero, tiene prioridad", dos hipótesis respecto a la asignación de las posiciones orbitales cuando sea necesaria la coordinación, teniendo en cuenta el principio de equi- dad y los países en desarrollo establecidos en la Convención de Nairobi de 1982 y en el artículo 44 de la Constitución de la uाt: "en caso de solicitudes equiparables para acceder al recurso órbita/espectro por parte de un país que ya tenga acceso a dicho recurso y un país en desarrollo u otro país que trate de acceder a él, el país que ya tenga ese acceso debe adoptar todas las medidas viables para permitir que el país en desarrollo o el otro país tenga acceso equitativo al recurso órbita/espectro solicitado". Es decir, que cuando exista un enfrentamiento por una posición orbital entre un país que ya accedió y un país que no ha accedido, o entre un país desarrollado y un país en vía de desarrollo debe evitarse el proceso de coordinación y dar registro a la posición orbital al país que no ha accedido o al país en vía de desarrollo.

\section{MARCO JURÍDICO Y EL PROBLEMA DE LA SOBERANÍA}

\section{A. La falta de delimitación del espacio aéreo y la costumbre internacional}

Aldo Armando Cocca en su libro Teoría del derecho interplanetario, escribe que "el primer problema jurídico importante que ha planteado el lanzamiento de un satélite artificial de la Tierra ha sido el que concierne a la soberanía de los Estados que violaría en su recorrido" (Rubio, 1954). Pues bien, aún hoy, después de 45 años de haberse aprobado el Tratado del 67 conocido como la "Constitución del Espacio", no existe tratado, convenio, declaración o acuerdo que haya definido el concepto de espacio ultraterrestre y menos fijado el límite en el espacio 
aéreo. Si bien los convenios internacionales de París y Chicago atribuyeron a las partes contratantes la soberanía sobre el espacio atmosférico situado sobre sus territorios, como en ellos no se prescribe de manera concreta, cifrada o de otro modo el límite altimétrico de la atmósfera, el alcance vertical de la soberanía resulta desconocido (Rubio, 1954).

Sin embargo, han surgido muchas hipótesis y propuestas que se fundamentan en consideraciones físicas, como la teoría de la división de la atmósfera según la cual el espacio ultraterrestre comenzaría cuando culmina esa capa; la teoría del efecto de la gravedad, criticable en cuanto la fuerza gravitatoria de la Tierra llega a los 256 mil y 1 millón 500 mil kilómetros; la teoría del espacio aéreo navegable, que afirma que el espacio exterior empieza entre los 80 y 100 $\mathrm{km}$, punto donde termina la reacción del aire (fuerza aerodinámica) y el vuelo solo es posible en virtud de la fuerza centrífuga (efecto Kepler), (Ramírez, 1958).

En medio de estas discusiones, en 1976 un representante del Comité de Investigaciones Espaciales (CIE), organización internacional que tiene el carácter de observadora ante la Comisión del Espacio Ultraterrestre, ofreció como solución la mayor altitud a la que los satélites se pueden movilizar todavía libremente sin ser obligados a bajar a tierra por corrientes de aire. Para satélites de órbitas circulares, la altura es de $130 \mathrm{~km}$ y para satélites en órbitas muy elípticas la altura aproximada es de $100 \mathrm{~km}$. En este sentido, se recomendó fijar el límite inferior del espacio ultraterrestre en 100 km, posición que fue aceptada por la mayoría de países (Ramírez, 1985).

\section{B. La Declaración de Bogotá}

Amparándose en la falta de delimitación del espacio ultraterrestre en algún convenio o tratado, y haciendo caso omiso de las propuestas y teorías científicas y técnicas, Colombia reivindicó en 1975, ante la Asamblea General de las Naciones Unidas, su soberanía sobre la órbita geoestacionaria que supra yace su territorio. Si bien su reclamo no fue acogido favorablemente por la Asamblea General de la onu, propició la reunión en Bogotá, entre el 29 de noviembre y 3 de diciembre de 1976, de los países sobre los cuales se encuentra el $30 \%$ de los segmentos de la órbita geoestacionaria o países ecuatoriales, a saber: Gabón, Zaire ${ }^{3}$, Uganda, Kenya y Somalia por África; Indonesia por Asia; Brasil (como observador), Ecuador y Colombia por América Latina (Ramírez, 1958), encuentro que culminó con la firma de la Declaración o acta de Bogotá, en la cual fue propuesto un régimen especial para los segmentos de la órbita geoestacionaria situados sobre sus respectivos territorios (Gaviria, 1958):

1. la órbita geoestacionaria se considera un recurso natural, sobre cuyos segmentos los Estados ecuatoriales pueden ejercer soberanía:

Los segmentos de la órbita sincrónica geoestacionaria son parte del territorio sobre el cual los estados ecuatoriales ejercen su soberanía nacional. La órbita geoestacionaria es un recurso natural escaso, cuya importancia y valor aumentará rápidamente junto con el desarrollo

3 Con este nombre fue conocido entre el 27 de octubre de 1971 y el 17 de mayo de 1997 el país africano actualmente llamado República Democrática del Congo. 
de la tecnología espacial y con la creciente necesidad de comunicación, por lo que los países ecuatoriales reunidos en Bogotá han decidido proclamar y defender en nombre de sus pueblos, la existencia de su soberanía sobre este recurso natural.

2. los segmentos más allá de la jurisdicción nacional de los Estados deben considerarse como "patrimonio común de la humanidad":

“Los segmentos de la órbita correspondiente a la mar abierto son más allá de la jurisdicción nacional de los Estados será considerado como patrimonio común de la humanidad. En consecuencia, los organismos internacionales competentes deben regular su uso y aprovechamiento en beneficio de la humanidad".

3. se exige la autorización previa y expresa del Estado ecuatorial respectivo, cuando se pretenda ubicar artefactos en los segmentos de su órbita geoestacionaria:

"Los productos a ser colocados permanentemente en el segmento de una órbita geoestacionaria ecuatorial de un Estado requerirá anterior y expresó su autorización por parte del Estado interesado, y el funcionamiento del dispositivo debe ser conforme con la legislación nacional de dicho país a lo largo territorial que se coloca".

4. los satélites que ya se encuentren en sus segmentos de órbita requerirán de un derecho de colocación expresamente autorizado por el Estado ecuatorial:

"Los estados ecuatoriales no toleran los actuales satélites o la posición que ocupan en sus segmentos de la órbita geoestacionaria ni la existencia de dichos satélites confiere ningún derecho de la colocación de satélites o el uso de la serie de sesiones a menos que sea expresamente autorizado por el Estado que ejerce su soberanía sobre este segmento".

A pesar de que la tesis de los países ecuatoriales fue rechazada por los países desarrollados y subdesarrollados, argumentando que violaba el principio de no apropiación, y que en igual sentido se pronunció la Conferencia Mundial de Radiotelecomunicaciones en Ginebra en 1978, posteriormente en la Conferencia de Nairobi de 1982 se firmó el Convenio Internacional de las Telecomunicaciones cuyo artículo 33 numeral 2 establece:

En la utilización de bandas de frecuencias para las radiocomunicaciones espaciales, los Miembros tendrán en cuenta que las frecuencias y la órbita de los satélites geoestacionarios son recursos naturales limitados que deben utilizarse en forma eficaz y económica, de conformidad con lo establecido en el Reglamento de Radiocomunicaciones, para permitir el acceso equitativo a esta órbita y a esas frecuencias a los diferentes países o grupos de países, teniendo en cuenta las necesidades especiales de los países en desarrollo y la situación geográfica de determinados países. (Subrayado fuera del texto)

Frente a esta última declaración, Colombia realiza una reserva entendiendo que por la situación geográfica especial de determinados países el artículo se refiere a los países ecuatoriales, a lo cual los países restantes responden con una contra reserva aclarando que no interpretan el artículo como lo entiende Colombia. 
Durante doce años los países ecuatoriales que firmaron la Declaración de Bogotá defendieron su posición pero gradualmente fueron abandonando sus reivindicaciones de soberanía en las Naciones Unidas, y en 1988 Ecuador era el único país que mantenía sus pretensiones ${ }^{4}$.

\section{Las Constituciones de Ecuador y Colombia}

La Constitución de Ecuador en su artículo 4, inciso 4 determina: "El Estado ecuatoriano ejercerá derechos sobre los segmentos correspondientes de la órbita sincrónica geoestacionaria, los espacios marítimos y la Antártida". (Destacados fuera del texto)

Este reclamo de soberanía es nulo hasta el momento si se tiene en cuenta que Ecuador poseía solamente un satélite de teleobservación (el NEE01 Pegaso, lanzado el 25 de abril del 2013 y que dejó de transmitir en mayo del mismo año después de colisionar con basura espacial), que por sus funciones se localizó en órbita baja y no tenía, en consecuencia, vocación de ejercer ningún derecho sobre la órbita geoestacionaria aun si no existiera el principio de no apropiación.

En el caso de Colombia, aunque el país abandonó su pretensión de soberanía en el seno de las Naciones Unidas, la Constitución de 1991 en su artículo 101, inciso 4 dispone:

4 Los últimos países que abandonaron esta posición fueron Colombia, Indonesia y Kenia, quienes ahora piden un "acceso equitativo" a esta órbita. Declaration of the first meeting of equatorial countries, reproducido en Böckstiegel H., Benkö M., Hobe S., Space Law Basic Legal documents, 2008, Vol. 2, B.IV.1.
También son parte de Colombia, el subsuelo, el mar territorial, la zona contigua, la plataforma continental, la zona económica exclusiva, el espacio aéreo, el segmento de la órbita geoestacionaria, el espectro electromagnético y el espacio donde actúa, de conformidad con el Derecho Internacional o con las leyes colombianas a falta de normas internacionales. (Destacados fuera del texto original).

De este artículo se desprende que así como lo hizo Ecuador, el reclamo de soberanía no es sobre toda la columna de aire y espacio que suprayace el territorio colombiano sino solamente sobre el segmento de la órbita geoestacionaria, considerado como un espacio aparte. Este artículo hace una aclaración importante y es que la reivindicación de soberanía debe sujetarse a las normas de derecho internacional, razón por la cual resulta inaplicable en atención a que el principio de no apropiación del espacio y los cuerpos celestes ha de ser respetado por Colombia como norma de Costumbre Internacional como se verá más adelante.

\section{Pronunciamientos de la Corte Constitucional colombiana}

La primera sentencia que fija su atención en el problema de la soberanía sobre el segmento de la órbita geoestacionaria es la C-382/96, con ponencia del magistrado José Gregorio Hernández Galindo. En ella se discute la viabilidad de un proyecto de ley que adopta la reglamentación sobre telecomunicaciones de la uIT, a la luz de la soberanía proclamada en el artículo 101 de la Constitución. La Corte estableció que no se niega la soberanía al firmar y ratificar el tratado de telecomunicaciones pues este solo 
ayuda a ampliar las relaciones internacionales, mandato de la Constitución. Además, establece que "Por otra parte, no es posible que las autoridades colombianas, ni administrativas ni judiciales, ejerzan en el orden interno las mencionadas facultades para interceptar o interrumpir comunicaciones de particulares con base en razones de Estado como las previstas, pues ello vulnera la inviolabilidad de la correspondencia e implica notorio abuso de quien ejerce el poder público" 5 . De esta forma, establece que no es posible ejercer la soberanía de la órbita de los satélites geoestacionarios pues no existe la tecnología ni los medios para ello, además de que se estarían violando otras normas.

Posteriormente, en la sentencia C-278 de 2004, con ponencia del magistrado Jaime Araújo Rentería, se habla específicamente de la soberanía sobre la órbita de los satélites geoestacionarios: "El texto de las normas precedentes [artículos 101 y 102] permite colegir que Colombia ejerce soberanía sobre el segmento de órbita geoestacionaria, en las mismas condiciones en que lo hace respecto del subsuelo, el mar territorial, (...)". No obstante, de la lectura detenida del artículo 101 se tiene que la órbita geoestacionaria es parte del territorio colombiano, “de conformidad con el derecho internacional o con las leyes colombianas a falta de normas internacionales", de lo cual se deduce que la normatividad del derecho internacional no es irrelevante para verificar el ejercicio de la soberanía nacional sobre dicha órbita.
Esta sentencia abre la posibilidad de estudiar la viabilidad de reivindicar soberanía teniendo en cuenta las normas del derecho internacional, entre las cuales se encuentra la Costumbre Internacional. Pero así como da este paso, se abstiene de reconocer el principio de no apropiación del espacio ultraterrestre, al establecer que "el Estado colombiano reafirma que el segmento de la órbita geoestacionaria que le corresponde forma parte del territorio colombiano según lo establecido en los artículos 101 y 102 de la Constitución, y entiende que ninguna norma de estas enmiendas es contraria a los derechos reclamados por los Estados ecuatoriales al respecto, ni podrá ser interpretada en contra de tales derechos." Afirmación que no es coherente con el principio de no apropiación que debe respetar Colombia como país firmante de la Convención de Viena de 1969 ni con los requisitos del derecho internacional para ejercer soberanía, ya que aun haciendo caso omiso del principio de no apropiación, el hecho de no tener satélites le impide ejercer soberanía sobre la órbita. Además, sobre el segmento de la órbita que suprayace el territorio colombiano se encontraban, para el año 2012, los satélites (vía satelital, 2013) Brasilsat-B3 $\left(75.0^{\circ} \mathrm{W}\right)$, de Brasil; Galaxy-9-spare $\left(74.9^{\circ} \mathrm{W}\right)$, Horizons-2 $\left(74.0^{\circ} \mathrm{W}\right)$, Directv- $1 \mathrm{R}$ $\left(72.5^{\circ} \mathrm{W}\right)$ y Americom-6 $\left(72.0^{\circ} \mathrm{W}\right)$, de Estados Unidos; Nahuel-1 $\left(71.8^{\circ} \mathrm{W}\right)$, operado en un $70 \%$ por Argentina, Chile, Uruguay y Brasil; y Star One C2 $\left(70.0^{\circ} \mathrm{W}\right)$, de Brasil y parcialmente de Luxemburgo, sin que Colombia haya solicitado su retiro o tomado acciones para evitar la "invasión" a su soberanía.

En la misma línea argumentativa, en la sentencia C-779/04, M. P.: Jaime Araújo Rentería, la 
Corte explica que el acceder a las regulaciones de la uit no implica renunciar a la soberanía sobre la órbita de los satélites geoestacionarios: “De esta manera, la regulación contenida en el artículo 44 de la Constitución de la uIT sobre la órbita geoestacionaria en manera alguna puede entenderse como una renuncia del Estado coIombiano a los derechos que sobre ella tiene, puesto que dicha interpretación iría en contra de lo prescrito por la Carta en los artículos 101 y $102 "$.

A partir de lo anterior, puede verse que la Corte Constitucional colombiana no ha querido adoptar una posición definitiva y ha buscado respuestas diplomáticas en las que, a la vez que hace un llamado a no desconocer las normas internacionales se niega a renunciar a las pretensiones de soberanía, contradiciendo así mismo la posición de Colombia de renunciar a los reclamos de soberanía en el marco internacional y optar por la búsqueda de un trato.

El que Colombia no adopte una política clara insinúa cierto temor a no poder tener una posición orbital privilegiada cuando esté en condiciones de acceder al espacio, teniendo en cuenta la ocupación que se viene dando por parte de los países desarrollados y que va más allá de la tesis de la soberanía. Temor que no es infundado a juzgar por las ya mencionadas disposiciones del Acuerdo de Constitución de la Unión Internacional de Telecomunicaciones que advierten sobre la necesidad de tener en cuenta a dichas naciones en la distribución de la órbita geoestacionaria, y que fueron aceptadas por Colombia mediante la aprobación de la Ley 252 de 1995, revisada y declarada exequible-con algunas ex- cepciones- por la Corte Constitucional, mediante la sentencia C-382 de 1996.

\section{E. El Tratado de 1967 y los principios de No Apropiación y Cooperación Internacional}

El Tratado del 67 sobre los principios que deben regir las actividades de los Estados en la explotación y utilización del espacio ultraterrestre, incluso la Luna y otros cuerpos celestes, consta de 17 artículos en los cuales se desarrollan diferentes preceptos, entre los cuales encontramos los siguientes: 1. Libertad de exploración; 2. Libertad de acceso; 3. No apropiación; 4. Uso pacífico; 5 . No utilización de armas nucleares; 6 . Permisividad de utilización de energía nuclear con fines pacíficos; 7. Responsabilidad por daños causados por objetos; 8. Propiedad y regulación de jurisdicción, control y devolución de objetos; 9. Cooperación Internacional; 10. Difusión de información. Sin embargo, para efectos del presente trabajo se tratarán los principios relativos a la no apropiación y la cooperación internacional, en el entendido que son pertinentes para defender la tesis según la cual no es posible reclamar soberanía sobre la órbita geoestacionaria o alguno de sus segmentos.

El principio de no apropiación del espacio ultraterrestre se encuentra consagrado en el artículo Il del Tratado sobre los principios que deben regir las actividades de los Estados en la exploración y utilización del espacio ultraterrestre, incluso la Luna y otros cuerpos celestes de 1967 que determina: "El espacio ultraterrestre, incluso la Luna y otros cuerpos celestes, no podrá ser objeto de apropiación nacional por reivindicación 
de soberanía, uso u ocupación, ni de ninguna otra manera. (Subrayado propio).

Una de las razones que fundamenta esta afirmación está relacionada con la órbita de los satélites geoestacionarios, ya que al ser esta un recurso natural limitado cuyo uso debe ser eficaz y económico tal y como dispuso el Tratado de Málaga-Torremolinos de 1973, no puede ser objeto de apropiación en la medida en que no es posible bajo supuestos de igualdad de acceso y de espacio distribuirla equitativamente; además, si se atendiera el reclamo de soberanía sobre su segmento de órbita geoestacionaria por parte de los países que no tienen la tecnología suficiente para acceder a ella, se impediría su uso a aquellos que sí están en capacidad de hacerlo y de ofrecer servicios a la humanidad, con lo cual se desatendería el requisito de eficacia. Hemos de recordar que "este es un principio revolucionario que en la ciencia del Derecho no tiene precedentes. Sin embargo, no podemos aceptar las afirmaciones de algunos internacionalistas en el sentido de que este principio viene del Tratado Antártico. Y ello es así porque la solución del Tratado Antártico es solamente un statu quo" (Ferrer, 1976). Por tanto, no puede pretenderse buscar su origen en el derecho internacional general, este principio corresponde a una disciplina altamente especializada correspondiente al derecho espacial.

Cabe anotar que este principio prohíbe la apropiación no solo por reivindicación de soberanía sino también por uso u ocupación, de tal forma que el poseer objetos en el espacio no da a la nación propietaria de ellos posibilidad de apropiarse de algún punto concreto, siendo así que "La norma internacional, así conformada, constituye fundamento del principio del espacio ultraterrestre como res communis omnium, o bien de toda la humanidad"6.

A diferencia del principio de no apropiación, el segundo principio (cooperación internacional) ha sido implementado en otras áreas del derecho internacional como en el Tratado Antártico, “con el que se habían establecido los principios del régimen jurídico de la exploración científica de la Antártida sobre la base de la experiencia adquirida durante el Año Geofísico Internacional”, evento que llamó la atención sobre la importancia de la exploración espacial para el desarrollo científico de cuestiones que involucraban a la humanidad, generándose la necesidad de la cooperación internacional en la búsqueda de dichos objetivos que beneficiaban a todos los habitantes del planeta (Kopal, 2009).

El principio de cooperación internacional en las actividades espaciales se encuentra consagrado en diferentes artículos del Tratado del 67 y sus consideraciones, las cuales implantan lo siguiente:

"Deseando contribuir a una amplia cooperación internacional en lo que se refiere a los aspectos científicos y jurídicos de la exploración y utilización del espacio ultraterrestre con fines pacíficos,

Estimando que tal cooperación contribuirá al desarrollo de la comprensión mutua y al afianzamiento de las relaciones amistosas entre los Estados y pueblos". (Subrayados propios fuera de texto).

6 
Específicamente, el Artículo III menciona este principio como guía para la aplicación del Derecho Internacional y la Carta de las Naciones Unidas a las actividades espaciales realizadas por los Estados Parte. Y el Artículo IX añade que estos últimos deben tener presentes los intereses correspondientes de los demás Estados Partes, en todas las actividades relacionadas con el espacio ultraterrestre. Es en este último punto donde se encuentra que todos los Estados tienen el deber de respetar y cooperar con los objetivos de los demás países, acudiendo a la colaboración e intercambio para la consecución de fines más altos los cuales son de la humanidad.

Este último principio se encuentra así mismo consagrado en el Tratado de Nairobi de 1982 el cual pretendió "facilitar las relaciones pacíficas, la cooperación y el desarrollo económico y social entre los pueblos, por medio del buen funcionamiento de las telecomunicaciones" añadiendo en su artículo 33 anteriormente mencionado el deber de los Estados de utilizar eficaz y económicamente la órbita geoestacionaria en atención a su carácter de recurso natural limitado.

\section{F. La Convención de Viena de 1969}

Si bien Colombia en su momento NO ratifica el Tratado de 1967, su vinculación a este se da por el artículo 38 de la Convención de Viena de 1969 (aprobada por la Ley 32 de 1985) que dispone: "Artículo 38. Normas de un tratado que Ileguen a ser obligatorias para terceros Estados en virtud de una costumbre internacional.
Lo dispuesto en los artículos 34 a 37 no impedirá que una norma enunciada en un tratado llegue a ser obligatoria para un tercer Estado como norma consuetudinaria de derecho internacional reconocida como tal".

De esta manera hemos de remitirnos al concepto de costumbre internacional. Según la concepción tradicional del proceso consuetudinario, la costumbre es el resultado del juego entre un elemento material (la práctica) y un elemento subjetivo (la opinio iuris), de acuerdo con lo siguiente: el comportamiento reiterado, constante y duradero de los Estados ante situaciones de relevancia internacional generaría, en un momento determinado, la convicción en los mismos acerca de su obligatoriedad, convirtiendo en exigencia normativa tal pauta de comportamiento (Fernández, 2011).

En derecho internacional, la adquisición de nuevos territorios por los miembros de la comunidad internacional se basa en la "Costumbre Internacional", una norma que señala que los Estados pueden reclamar soberanía sobre territorios que no pertenecen a nadie (Terra NuIlius). Es así que en 1967, ante el riesgo de que los Estados pudieren reclamar soberanía sobre la Luna y otros cuerpos celestes y todo lo contenido en el espacio ultraterrestre, diferentes países firmaron y ratificaron o solo firmaron el Tratado de 1967 (Mejía, 2008) consagrándose el principio de no apropiación en el espacio. De esta manera, los Estados que han firmado este Tratado se han obligado a no aplicar la costumbre internacional en la adquisición de nuevos territorios más allá de la Tierra. 
La posición de Colombia sobre el tema de la órbita geoestacionaria fue claramente expuesta por Diego Pardo Tovar en el Simposio Internacional sobre Órbita Geoestacionaria, organizado por el CIDA-E en 1980, en el que su contraparte fue Manuel Augusto Ferrer (tratadista de derecho aeronáutico y espacial).

En aquella oportunidad, Pardo Tovar sostuvo que todo el segmento de la órbita geoestacionaria de satélites que surcan el cielo territorial colombiano, entre los grados 70 y 75 al Oeste de Greenwich, era considerado por el país como un "recurso natural", constituyendo un bien inalienable e imprescriptible de la Nación, a cuya soberanía no podría renunciar a tenor de su Constitución.

Colombia, decía Pardo Tovar, considera que el principio mencionado en el art. II del Tratado del Espacio de 1967, según el cual "el espacio ultraterrestre, incluso la Luna y otros cuerpos celestes, no podrá ser objeto de apropiación nacional por reivindicación de soberanía, uso u ocupación, ni de ninguna otra manera", es aplicable a la órbita geoestacionaria por no existir una definición del espacio ultraterrestre. Además, al no haber Colombia ratificado el Tratado del Espacio, sus disposiciones no podían invocarse en su contra.

Ferrer, por su parte, fundamentó la posición contraria, señalando que la pretensión de reclamar soberanía hacia arriba por proyección vertical de los derechos de soberanía, era un absurdo cosmográfico. Consideró inaceptable la Declaración de Bogotá de 1976 por contrariar principios vigentes en la comunidad internacional. Ferrer afirmaba que si bien hay países que no son par- te del Tratado del Espacio, hay principios como el de la libertad de tránsito y de no apropiación nacional del espacio ultraterrestre, que son normas de derecho internacional positivo, que revisten carácter de normas de jus cogens.

Todos los países que firmaron la Declaración de Bogotá, decía Ferrer, han aprobado por unanimidad diversas resoluciones de las Naciones Unidas que establecen esos principios y que muchos consideran que generan una "costumbre internacional instantánea". Si bien Ferrer reconoce que dichas Resoluciones no son tratados debidamente ratificados, sostiene que tienen esa general aceptación denominada "communis oppinio" (Gaggero, 2013). Cumpliéndose de esta manera el requisito de la existencia de un opinio iuris.

Aunado a lo anterior, se tiene que como hemos visto, los únicos dos países que poseen reivindicaciones de soberanía sobre la órbita geoestacionaria en sus Constituciones son Colombia y Ecuador, y que estas son disposiciones débiles pues, por un lado, Ecuador no ha ejercido derechos sobre la geo y la disposición de Colombia se encuentra condicionada al cumplimiento de normas internacionales entre las cuales está la costumbre internacional. En el mismo sentido, reiterándose el requisito de la práctica, ninguno de los Estados que han accedido al espacio ultraterrestre han reclamado soberanía y se han opuesto a ello. La nueva costumbre internacional de no apropiación de nuevos territorios en el espacio y los cuerpos celestes es entonces obligatoria para todos los Estados de la comunidad internacional hayan o no ratificado el Tratado del espacio (Gaggero, 2013). 
La Convención de Viena sobre el derecho de los tratados, en su artículo 53, establece que una norma imperativa o de ius cogens es aquella que es aceptada y reconocida por la comunidad internacional, que no admite acuerdo en contrario y que solo puede ser modificada o derogada por una norma que presente el mismo carácter. En este sentido, debe tratarse de normas que incorporen valores fundamentales para la comunidad internacional y que supongan un límite a la autonomía de la voluntad de los Estados. Para muchos, el principio de la cooperación internacional cumple estas características (Lacleta, 2005) y, por ende, en virtud de este principio los reclamos de soberanía sobre la órbita geoestacionaria no serían viables puesto que contrariarían una norma de tal nivel que, como hemos visto, implica la necesidad de realizar un uso eficaz y económico de dicha la órbita.

Además, así como el artículo 38 de la Convención de Viena de 1969 reconoce que la eficacia relativa de los tratados no excluye la obligatoriedad para terceros de las normas en él contenidas que ostenten o adquieran naturaleza consuetudinaria, según el artículo 43 el que un tratado deje de estar en vigor para un Estado como consecuencia de su nulidad, terminación o denuncia, no afecta su obligación de cumplir las disposiciones de este dotadas de valor consuetudinario. Igualmente, habrá que entender que una norma consuetudinaria no resulta afectada en cuanto a su carácter obligatorio por el hecho de que la disposición convencional que la incorpora sea objeto de reservas por un Estado o, incluso, porque se encuentre recogida en un convenio todavía no en vigor (Fernández, 2011).
Adicionalmente, ya en la decisión de la Corte Internacional de Justicia sobre el caso de la Plataforma Continental en el Mar del Norte, esta indicó que una norma de derecho internacional de Costumbre, que se originó a partir de una provisión de un tratado internacional, se transforma en obligatoria aun para los países que no han formado parte de ese tratado.

Si bien existe la figura del "objetor persistente", por la cual cuando un Estado se opone a una práctica que es seguida y reconocida por varios miembros de la comunidad internacional, al esta convertirse en Costumbre Internacional no le es aplicable, no puede decirse que Colombia encaje dentro de esta descripción ya que:

1. Dejó de reivindicar su soberanía sobre la GEO en foros internacionales.

2. Aunque adoptó de nuevo la tesis de la soberanía en su Constitución, no ha exigido a los dueños de los satélites ubicados en su presunto segmento de la GEO que retiren los objetos espaciales.

3. No ha denunciado a los supuestos transgresores de su pretendida soberanía.

4. Mantiene contratos para servicios satelitales con compañías privadas extranjeras que usan "su" segmento de la órbita.

5. No posee satélites geoestacionarios.

Todas estas inconsistencias hacen que sea aplicable la costumbre internacional a Colombia y a Ecuador (2-5) por reunir las mismas cualidades, negándoles la oportunidad de aducir objeción persistente para la no aplicación del principio 
de no apropiación consagrado en el Tratado de 1967.

\section{PROPUESTA PARA EL USO DE LAS ÓRBITAS}

A través del estudio del espacio y del espaciotiempo se ha llegado a los grandes triunfos contemporáneos en el campo de la energía atómica, y por ellos también a las grandes exploraciones, aún incipientes, del espacio, las cuales no deben ser ajenas al hombre de leyes, al derecho en sí, a la ley (Estradé, 1964).

Por consiguiente y considerando:

1. Que el carácter de patrimonio común de la humanidad revestido por la órbita geoestacionaria ha sido reconocido.

2. Que el Tratado Málaga-Torremolinos de la uाт fue modificado en 1982 por la reunión de Nairobi de dicha institución, donde se agrega la necesidad de respetar la situación geográfica de algunos países.

3. Que el reconocimiento de una jurisdicción o privilegios, por limitados que sean, en materia de órbita geoestacionaria entraría en conflicto directo con los principios jurídicos que rigen las actividades en el espacio ultraterrestre (Williams, 1990).

4. Que según Naciones Unidas (1994) Francia, en 1989, en el marco de sus propuestas sobre la inmunidad de los satélites se centró en la formulación de normas de buena conducta para los vehículos espaciales, con miras a reducir el riesgo de colisiones accidentales, prevenir las persecuciones coorbitales a corta distancia y garantizar un mejor conocimiento del tráfico espacial mediante: a) Disposiciones para actualizar de manera regular los elementos orbitales declarados en el momento del registro, en el caso de maniobras y derivas, b) El respeto de una distancia mínima entre dos satélites colocados en una misma órbita para evitar no solo las colisiones accidentales, sino también las persecuciones coorbitales a corta distancia, que son un requisito necesario para el sistema de minas espaciales, y c) La vigilancia de los cruces a corta distancia para limitar los riesgos de colisión o de interferencia.

5. Que el Tratado sobre los principios que deben regir las actividades de los Estados, de 1967, en su art. 9 dice que: "En la exploración y utilización del espacio ultraterrestre, incluso la Luna y otros cuerpos celestes, los Estados Partes en el Tratado deberán guiarse por el principio de la cooperación y la asistencia mutua", por lo que este artículo asienta el principio de cooperación internacional en cuanto a la actividad espacial a desarrollarse, por otro lado, el art. 10 suscribe a esta idea formulando que " $A$ fin de contribuir a la cooperación internacional en la exploración y la utilización del espacio ultraterrestre, incluso la Luna y otros cuerpos celestes, conforme a los objetivos del presente Tratado, los Estados partes en él examinarán, en condiciones de igualdad", esta normativa intenta integrar en pos de la igualdad a aquellos Estados que no tengan la misma oportunidad tecnológica debido a su economía, equiparándolos en sus condiciones en la parte final de dicho artículo: "La naturaleza de tal oportunidad y las condiciones en que podría ser concedida se determinarán por acuerdo entre los Estados interesados".

Se propone la realización de un régimen jurídico especial para la órbita geoestacionaria donde 
se tenga en cuenta que, si bien la soberanía no puede concebirse como absoluta por ser contraria a los progresos de la técnica y, en general, de la cultura humana, tampoco la libertad o facultad pueden ser absolutas sino para algo determinante, un fin que, conforme al estado de la cultura universal, pueda considerarse lícito y sin caer en abuso de derecho (Rubio, 1958).

De esta manera, se ponen a consideración las siguientes medidas:

1. La creación de un fondo administrado por la UIT, el cual se alimentará del dinero aportado por aquellos países que deseen obtener una posición orbital y una frecuencia radioeléctrica y que ya posean un número superior de satélites a la media mundial.

2. La implementación de las propuestas realizadas por Francia en 1989 y las demás pertinentes, con el fin de evitar colisiones e interferencias entre satélites, tales como el llevar los satélites a órbitas altas y la utilización de las órbitas generalizadas.

3. La implementación de las propuestas realizadas por Colombia en el documento A/ AC.105/C.2/L.200, presentado por el doctor Alfredo Rey Córdoba ${ }^{7}$ en el año 2000, aún en consideración, por las cuales se daría aplicación a los principios de acceso equitativo y racional al ROE, de tal forma que cuando fuere necesaria la coordinación para establecer qué país tendrá acceso a determinada posición orbital y frecuencia radioeléctrica:

$7 \quad$ Cabe anotar que el doctor Rey concuerda en que se hace imposible realizar reivindicaciones de soberanía sobre la órbita de los satélites geoestacionarios. Sin embargo, aclara que así mismo es indudable que a los 35875 km sobre la línea del Ecuador se presenta un fenómeno único que debe ser analizado de manera individual y asignársele un régimen jurídico especial. a. Entre un país que ya tenga acceso y uno que no, se le dé prioridad al segundo.

b. Entre dos países que ya tengan acceso o dos países que no lo tengan se mantenga el principio de que "quien llega primero, tiene prioridad".

Estas propuestas fueron ampliadas por los estudiantes del curso de Derecho Espacial (primer periodo del 2013), dictado por el doctor Alfredo Rey en la Universidad de los Andes, teniendo en cuenta todas las posibles situaciones que pudieren presentarse al momento de necesitarse la coordinación entre diversos tipos de países, con las siguientes ideas:

a. Entre un país que ya accedió y uno que no ha accedido, se dé prelación al país que no ha accedido.

b. Entre un país que ya accedió y uno en vía de desarrollo, deberá tener prelación el país en vía de desarrollo sin necesidad de coordinación. Sin embargo, si este país en vía de desarrollo ya accedió a una posición orbital se deberá entrar a coordinar bajo el principio de "quien llega primero, tiene prioridad".

c. Entre un país que ya accedió y un país desarrollado debe operar el principio de "quien llega primero, tiene prioridad".

d. Entre dos países que ya accedieron aplicará el principio de "quien llega primero, tiene prioridad".

e. Entre un país que no ha accedido y un país en vía de desarrollo, si el país en vía de desarrollo ya accedió se debe dar prelación al país que no ha accedido. Si el país en vía de 
desarrollo no ha accedido se debe seguir el procedimiento de coordinación bajo el principio de "quien llega primero, tiene prioridad".

f. Entre un país que no ha accedido y un país desarrollado tendrá prelación el país que no ha accedido, sin necesidad de coordinación.

g. Entre dos países que no han accedido deberá darse el proceso de coordinación, bajo el principio de "quien llega primero, tiene prioridad". No obstante, si alguno de los dos es un país en vía de desarrollo deberá darse prioridad a este sin necesidad de coordinación.

h. Entre dos países en vía de desarrollo tendrá prioridad el que no ha accedido, sin necesidad de coordinación. De lo contrario se debe entrar a coordinar bajo el principio de "quien llega primero, tiene prioridad".

i. Entre un país en vía de desarrollo y un país desarrollado debe acceder el país en vía de desarrollo.

j. Entre dos países desarrollados debe aplicarse el principio de "quien llega primero tiene prioridad"

4. Hacer obligatoria la inscripción en el registro internacional de los objetos espaciales para que haya un mayor control de las posiciones reales de los satélites y de su órbita.

Así como los satélites en órbita geoestacionaria se envían a órbitas altas con el poco combustible que les queda, debieran traerse hacia la atmósfera a los satélites en órbitas bajas para que sean incinerados por la fricción del aire.
Promover la utilización de vehículos espaciales reutilizables cuyas partes vuelvan a la tierra, evitando la saturación de las órbitas y constituyendo un ahorro económico para los países.

\section{CONCLUSIONES}

1. La conquista espacial se ha vuelto un asunto de los que pueden y no de los que quieren o de los que tienen fundados sus derechos y necesidades, en manos de Estados desarrollados con poder económico y tecnológico (Comisión Colombiana del Espacio).

2. El sueño de la humanidad de aprovechar al máximo el espacio ultraterrestre para el desarrollo de la ciencia y el bienestar de la humanidad aún no se ha hecho realidad y, por lo tanto, sigue siendo un objetivo por alcanzar.

3. La delimitación y definición del espacio ultraterrestre reviste en este momento primera prioridad.

4. La altura a partir de la cual comienza el régimen de libertad de exploración y utilización consagrado por el Tratado del Espacio de 1967 e instrumentos afines podría ser fijada, por convención, en alrededor de los $100 \mathrm{~km}$, teniendo en cuenta que en la Resolución de Manila se declaró que el espacio situado más allá de los 100 km sobre el nivel del mar estaba considerado por un importante número de Estados y de la doctrina como espacio ultraterrestre.

5. La órbita geoestacionaria, al igual que toda órbita descrita por un satélite, se encuentra en el espacio ultraterrestre. Por ende, está sujeta 
a los principios de libre acceso y no apropiación aplicables en ese ámbito. Estos principios no resultan afectados por los sistemas de planificación que, por razones administrativas, establezcan las Conferencias de la uit (Williams, 1990).

6. El fenómeno natural de la órbita geoestacionaria solo aparece en el plano ecuatorial, lo cual constituye una realidad objetiva que la hace única en su naturaleza. Por otra parte, la órbita geoestacionaria es un recurso natural limitado por cuanto se puede llegar a saturar y se hace necesaria una regulación especial.

7. Los satélites geoestacionarios no están ubicados en posiciones fijas de manera permanente. A lo largo de sus vidas activas se deben mantener en una región en torno de sus posiciones nominales, mediante sistemas de propulsión de mantenimiento.

8. Para tener la soberanía sobre algún tipo de territorio es necesario poder ejercerla, y como bien lo estableció la Corte Constitucional de Colombia en 1996, es imposible para Colombia defender la órbita de los satélites geoestacionarios si, hipotéticamente, alguien llegara a violar su soberanía.

9. Una de las bases sobre la que se construyó el derecho del espacio es su no apropiación. Por más que Colombia no haya ratificado el Tratado de 1967 sino hasta hace muy poco, no podía ignorar el hecho de que ningún país le iba a permitir reclamar soberanía sobre el espacio, ya que por la Convención de Viena se aplica la costumbre y esta dictamina que el espacio no es libre de apropiación.
10.No es posible la apropiación del espacio ultraterrestre. No existe duda de que a una altura de $36.000 \mathrm{~km}$, la órbita geoestacionaria se encuentra en el espacio ultraterrestre. Todo reclamo de soberanía más allá de los 100/110 $\mathrm{km}$ iría en contra de los principios de libertad de utilización y no apropiación, teniendo en cuenta que ya se ha formado una costumbre internacional con respecto al punto en que nos encontramos en el espacio ultraterrestre. Además, las características y atributos físicos de la órbita dependen de la Tierra considerada como un todo, y en consecuencia no existe la base para que un grupo de Estados reclame soberanía, jurisdicción o derechos preferenciales. (Doc. A/AC. 105/370).

11. En el futuro es posible para los países en vía de desarrollo rescatar la preocupación por el acceso al espacio, ya que es claro que los países desarrollados tienen una ventaja muy grande sobre estos en materia de derecho espacial.

\section{Bibliografía}

Abourdaham, P. (2001). Junta del reglamento de radiocomunicaciones. Recuperado desde: http://www.itu.int/itunews/issue/2001/10/ rrb-es.html.

Böckstiegel, H., Benkö, M., Hobe, S. (2008). Space Law Basic Legal documents, Vol. 2, B.IV.1.

Buedeler, W. (1957). El año geofísico internacional. París: Organización de las Naciones Unidas para la Educación, la Ciencia y la Cultura. 
Comisión Colombiana del Espacio. (2011). Evolución del derecho espacial. Recuperado desde: http://www.cce.gov.co/alfresco2.1-5.1.1.1/ d/d/workspace/SpacesStore/33735426d997-11e0-839f-c35ee8efbcbe/7.Yinet\%20 Cordoba,\%20VICEPRESIDENCIA.pdf.

CNES. (1996). El espacio: ¿cómo funciona?, ¿para qué sirve? Madrid: Proespacio

Estradé Rodoreda, S. (1964). El derecho ante la conquista del espacio. Barcelona: Ariel.

Ferrer, M. A. (1976). Derecho espacial. Buenos Aires: Plus Ultra.

Franco García, Á. (2010). Física con ordenador: dinámica celeste. Recuperado desde: http:// www.sc.ehu.es/sbweb/fisica/celeste/kepler3/kepler3.html, 2010

GagGero Montaner, M. (2013). Es necesario establecer el límite entre el espacio aéreo y el espacio ultraterrestre a la luz de los nuevos desarrollos tecnológicos? Asociación Latinoamericana de Derecho Aeronáutico y Espacial (ALADA). Recuperado desde: http://alada.org/ es-necesario-establecer-el-limite-entre-el-espacio-aereo-y-el-espacio-ultraterrestre-a-laluz-de-los-nuevos-desarrollos-tecnologicos/

Gaviria Liévano, J. E. (1978). Régimen jurídico de la órbita geoestacionaria y el espacio ultraterrestre. Bogotá: Universidad Externado de Colombia - ITU. Acerca de la UIT. Recuperado desde: http://www.itu.int/es/about/Pages/ history.aspx, 1978.
Kopal, V. (2009).Tratado sobre los principios que deben regir las actividades de los Estados en la exploración y utilización del espacio ultraterrestre, incluso la Luna y otros cuerpos celestes. Organización de las Naciones Unidas. Recuperado desde: http://untreaty.un.org/cod/avl/pdf/ha/ tos/tos_s.pdf

LACLeta Muñoz, J. M. (2005). El derecho en el espacio ultraterrestre. Real Instituto Elcano de Estudios Internacionales y Estratégicos. Recuperado desde: http://www.realinstitutoelcano. org/wps/portal/rielcano/contenido?WCM_ GLOBAL_CONTEXT=/elcano/elcano_es/zonas_es/defensa+y+seguridad/dt18-2005.

Medina Velandia, L. (2012). Tipos de órbitas. Bogotá: Universidad Sergio Arboleda. Recuperado desde: http://ingenierias.usergioarboleda.edu.co/index. php?option $=$ com_k2\&view=item\&id=742: tipos-de\%C3\%B3rbitas\&ltemid=280.

MejíA, M. (2008). Propiedad privada y soberanía en el espacio. México: Biblioteca Jurídica Virtual del Instituto de Investigaciones Jurídicas de la unam. Recuperado desde: http://biblio. juridicas.unam.mx/libros/6/2790/9.pdf.

NACIONES Unidas. Centro para el Desarme. (1994). Estudio sobre la aplicación de medidas de fomento de la confianza en el espacio ultraterrestre. New York: Naciones Unidas.

Naciones UniDAs, Asamblea General. (1977). Carácter físico y atributos técnicos de la órbita geoestacionaria (A/AC.105/203). New York: Naciones Unidas. 
Peredo Álvarez, S. (2004). Software para análisis del presupuesto de enlace para comunicaciones vía satélite: Cap. II Satélites. Recuperado desde: http://catarina.udlap.mx/u_ dl_a/tales/documentos/lem/peredo_a_s/ capitulo2.pdf.

Ramírez Del Valle, B. (1985). La órbita sincrónica geoestacionaria: tercera dimensión de la soberanía nacional. Cartagena: Centro de Investigaciones Jurídicas Unicartagena.

Rubio Tardío, P. (1958). Derecho y satélites artificiales: más sobre la naturaleza jurídica del espacio supraterrestre. Madrid: Instituto Francisco de Vitoria.

Salvat editores. (1974). Los satélites artificiales. Barcelona: Salvat.

Seara Vázquez, M. (1980). Derecho y política en el espacio cósmico. Mexico: Unam. Instituto de Investigaciones Jurídicas.

TRENCH, M. V. (2011). El derecho espacial y la cooperación internacional ante los desastres naturales. Recuperado desde: http://www. derecho.uba.ar/institucional/deinteres/derecho-internacional-publico-mariana-trench. pdf.

Vía Satelital. (2012). Lista de satélites en órbita geoestacionaria - hemisferio Occidental. Recuperado desde: http://www.viasatelital. com/satelites/.

Williams, S. M. (1990). Derecho internacional contemporáneo: la utilización del espacio ultraterrestre. Buenos Aires: Abeledo-Perrot.

\section{Convenios y Tratados}

Acuerdo que debe regir las actividades de los Estados en la Luna y otros cuerpos celestes (Resolución 34/68 de la Asamblea General, anexo), aprobado el 5 de diciembre de 1979, abierto a la firma el 18 de diciembre de 1979. Entró en vigor el

11 de julio de 1984.

Algunos aspectos relativos a la utilización de la órbita geoestacionaria, documento aprobado por la Subcomisión de Asuntos Jurídicos en su 39 periodo de sesiones (A/AC.105/738, anexo III).

Convenio sobre la responsabilidad internacional por daños causados por objetos espaciales (Resolución 2777 (XXVI) de la Asamblea General, anexo), aprobado el 29 de noviembre de 1971, abierto a la firma el 29 de marzo de 1972. Entró en vigor el 11 de septiembre de 1972.

Convenio sobre el registro de objetos lanzados al espacio ultraterrestre. Adoptado por la Asamblea General de la onv el 12 de noviembre de 1974. Resolución 3235 (XXIX). Entró en vigor el 15 de setiembre de 1976, de acuerdo con el artículo VIII, parágrafo 3.

Declaración sobre la cooperación internacional en la exploración y utilización del espacio ultraterrestre en beneficio e interés de todos los Estados, teniendo especialmente en cuenta las necesidades de los países en desarrollo, aprobada el 13 de diciembre de 
1996 (Resolución 51/122 de la Asamblea General).

Principios que han de regir la utilización por los Estados de satélites artificiales de la Tierra para las transmisiones internacionales directas por televisión, aprobados el 10 de diciembre de 1982 (Resolución 37/92 de la Asamblea General).

Principios relativos a la teleobservación de la Tierra desde el espacio, aprobados el 3 de diciembre de 1986 (Resolución 41/65 de la Asamblea General).
Tratado sobre los principios que deben regir las actividades de los Estados en la exploración y utilización del espacio ultraterrestre, incluso la Luna y otros cuerpos celestes (Resolución 2222 (XXI) de la Asamblea General, anexo), aprobado el 19 de diciembre de 1966, abierto a la firma el 27 de enero de 1967. Entró en vigor el 10 de octubre de 1967. 